\title{
Depth-Resolved Dynamics of Aceto-Whitening in Rabbit Cornea Studied ar 7300 nm Optical Coherence Tomography
}

DOI 10.17691/stm2015.7.1.07

Received November1, 2014

P, Khoo, MEng, Graduate, Department of Materials Science and Engineering';

S. Mittar, PhD, PDRA, Department of Materials Science and Engineering';

D. Kasaragod, PhD, PDRA, Department of Materials Science and Engineering'; Research Fellow²;

Z. Lu, PhD, PDRA, Department of Materials Science and Engineering';

Department of Electrical \& Electronic Engineering";

S.J. Matcher, PhD, Reader, Department of Materials Science and Engineering ${ }^{1,4}$

The Kroto Research Institute, University of Sheffield, North Campus, Broad Lane, Sheffield, S3 7HQ,

United Kingdom,

${ }^{2}$ Computational Optics Group, Institute of Applied Physics, University of Tsukuba, Tennodai 1-1-1, Tsukuba, Ibaraki,

305-8573, Japan;

${ }^{3}$ Nanoscience \& Technology Centre, University of Sheffield, North Campus, Broad Lane, Sheffield, S3 7HQ,

United Kingdom;

${ }^{4}$ Insigneo Institute for in silico Medicine: F Floor- Room F19, The Pam Liversidge Building, Sir Frederick Mappin Building,

The University of Sheffield, Mappin Street, Sheffield, S1 3JD, United Kingdom

In many developing parts of the world, colposcopy supplemented by aceto-whitening remains the most widespread means of reliably diagnosing cervical dysplasia, potentially leading to cervical cancer. The use of aceto-whitening to enhance detection rates for Barrett's esophagus is also emerging in recent years. Here we quantitatively study the aceto-whitening phenomenon using $1300 \mathrm{~nm}$ optical coherence tomography. By exposing a simple epithelial tissue model (excised rabbit cornea) to $0.5 \mathrm{M}$ acetic acid we report for the first time to our knowledge the direct measurement of the time over which the cellular brightening effect propagates down through the epithelial layer and into the stroma ( 50 s). We also measure the magnitude of the cellular brightening effect in quantified units $(\sim 10 \mathrm{~dB}$ in the epithelium). We also exposed the rabbit corneas to two control substances for clinical aceto-whitening, $0.5 \mathrm{M}$ lactic acid and phosphate buffered saline. Phosphate buffered saline produced no discernible change in the optical properties of the corneas. However we noted that lactic acid (an alpha-hydroxy acid) produced a pronounced brightening in the epithelial layer also, peaking at $8 \mathrm{~dB}$ after $20 \mathrm{~s}$. The main distinction with acetic acid was a lack of effect in the corneal stroma. This may point to a way of giving OCT imaging the ability to discriminate between cells of epithelial vs fibroblastic phenotype.

Key words: optical coherence tomography; aceto-whitening; acetic acid; lactic acid; cornea; epithelium; stroma.

Introduction. The last few decades have seen great strides in developing photonics-based imaging techniques for in vivo use in the human body. Many applications for these platforms exist, but a particularly compelling one is the early detection of dysplasia in epithelial tissue and transformation towards malignant and invasive cancer. Since over $80 \%$ of all primary tumours are estimated to arise in the epithelial tissue the limited depth penetration of photonic imaging is not a major disadvantage as the epithelium is frequently just a few hundred microns thick. Video rate confocal microscopy and endoscopic confocal microscopy have been shown to possess the potential to non-invasively detect changes in cell nuclear morphology, an established marker for dysplastic change [1, 2]. Optical coherence tomography (OCT) offers lower spatial resolution than confocal microscopy but a much higher voxel acquisition rate [3] and has also been shown to have potential in detecting areas of gross dysplastic change such as basal cell carcinoma nests [4]. Such techniques have the potential to be further developed towards tools for non-invasively determining tumour resection margins and for guiding the site of biopsy sampling to reduce false negatives when biopsying diffuse disease.

Despite the proven success of these techniques in providing label-free images nevertheless there is interest in further boosting the contrast by the use of exogenous substances. Established methods of modulating the absorption properties of tissue include non-specific dyes such as Lugol's iodine, touldine blue and acriflavine [5]. Plasmonic resonant gold nanoparticles have a proven ability to modulate both scattering and absorption contrast, depending on their size [6]. The relatively large size of gold nanoparticles that have a plasmon resonance in the nearinfrared has implications for the efficiency of topical delivery however.

It has been known for many years that screening for dysplasia in the female cervix using low-magnification microscopy (colposcopy) can be significantly enhanced by the topical spraying of 3-5\% acetic acid [7]. The interaction between cervical tissue and the weak acid is known empirically to result in a significant increase in optical opacity in areas that are dysplastic. This phenomemon is

Corresponding author: Stephen J. Matcher, e-mail: S.J.Matcher@sheffield.ac.uk 
known as the aceto-whitening effect (AWE). The precise cause of the AWE is somewhat controversial. MacLean [7] notes that AWE is not specific to acetic acid but can be produced by other carboxylic acids such as formic, propionic and butyric acid as well as by isomers of these. A concentration of at least $3 \%$ was reported as being necessary to produce pronounced and stable AWE in vivo. Other organic and inorganic acid solutions of similar $\mathrm{pH}$ but not from the carboxylic family do not produce the clinical AWE, in particular both citric and lactic acid (both alpha hydrox acids) are reported in the article (MacLean) to be negative controls for the clinical AWE. The origin of the AWE also does not seem to be dependent on the presence of an acetate group, as sodium acetate is a negative control for clinical AWE. MacLean suggests that AWE is associated with either the intracellular concentration of non-ionised acetic acid or the $\mathrm{pH}$; where the concentration of acid leads to conformational changes in cellular components such as denaturation of protein, fixation and cross-linking.

Maddox et al. [8] applied immunohistochemical staining to biopsy slides taken from areas of cervical tissue that displayed AWE and from areas not showing AWE. LH2-antibody labeling revealed an apparent correlation between cytokeratin-10 levels and the degree of AWE. Since cytokeratin-10 is a protein found in the intermediate filaments forming the cytoskeleton of epithelial cells, the authors speculated that cytokeratin-10 was an essential component of the AWE. Since this protein is found in epithelial cells but not fibroblastic cells (where vimentin assumes the cytoskeletal functions of the cytokeratins) this supports the theory that AWE originates in the epithelium. However recently Marina et al. [9] used flow cytometry at $785 \mathrm{~nm}$ to compare AWE in the epithelial cervical carcinoma cell line $\mathrm{SiHa}$ with that in the tumorigenic fibroblastic cell line MR1. Although the induced acetic acid concentration in the samples, $0.6 \%$, is arguably too low to replicate clinical conditions, nonetheless significant, and broadly similar, brightening was achieved for both cell types, suggesting that neither cytokeratin-10 nor human papilloma virus (not present in MR1 cells) is required for AWE to occur.

An improved understanding of the origins of the AWE is desirable, especially as the use of acetic acid begins to generate interest beyond colposcopy, into Gl screening for Barrett's esophagus [10] and gastric [11, 12] and oral cancer [13], for example.

Balas et al. [14] developed a detailed mathematical model of the AWE in cervical tissue, demonstrating that the model successfully predicts the timescale over which the clinical AWE occurs in dysplastic cervix peaks (50 s). The model assumes that the change in optical scattering correlates with the intracellular proton kinetics within the cervical epithelial cells. A key idea underpinning the model is that a substantial difference exists between the extracellular $\mathrm{pH}$ of dysplastic vs normal tissue, with the greater acidity of dysplastic tissue increasing the ratio of unionized to ionized acetic acid in the extracellular space. Since unionized acetic acid can diffuse across the cell membrane more readily than the ionized form, this results in higher intracellular acidity and hence proton concentration in dysplastic tissue. Detailed modeling suggests that the intracellular proton concentration peaks at between 30 and $150 \mathrm{~s}$ for CIN grades 1-3 respectively, before falling away as buffering mechanisms and active ion pumps restore baseline values. The decay is markedly slower for the higher grades. The model predictions are in excellent agreement with experimental measurements made using the DySIS digital colposcopy system [15]. A key feature of the model of Balas et al. is its inclusion of a realistic 3D layered epithelium through which the acetic acid is assumed to diffuse. The timescales of the diffusion through the epithelium and into the stroma thus become controlled by parameters such as the area of the tight junctions which physically couple neighboring epithelial cells.

The Balas model emphasizes the advantages of studying the AWE phenomenon in a realistic 3D geometry. Since experimental reports into the origins of AWE have tended to be performed on isolated cells or cultured monolayers, we decided to study the depth-resolved dynamics of acetowhitening in a simple 3D model system, the rabbit cornea.

The cornea (Figure 1) is the transparent front part of the eye that covers the iris, pupil, and anterior chamber. It accounts for approximately two-thirds of the eye's total optical power. The human cornea consist of five layers namely the epithelium and its basement membrane, Bowman's membrane, the stroma, Descement's membrane and the endothelium. Rabbit cornea has an approximate thickness of $410 \mu \mathrm{m}$ and is composed of four distinct layers, i.e. non-keratinised stratified squamous epithelial layer $(35-40 \mu \mathrm{m})$, corneal stroma, Descemet's membrane $(8-10 \mu \mathrm{m})$ and monolayer of endothelium (Descemet's mesothelium, 3.5-5 $\mu \mathrm{m}$ ) [16]. The Bowman's membrane is absent from the rabbit cornea [17, 18]. Laser scanning confocal microscopy established a tortuous basal lamina adjoining the epithelium of the rabbit cornea [19]. The underlying corneal stroma comprises about $90 \%$ of the total thickness of rabbit cornea. In addition a high

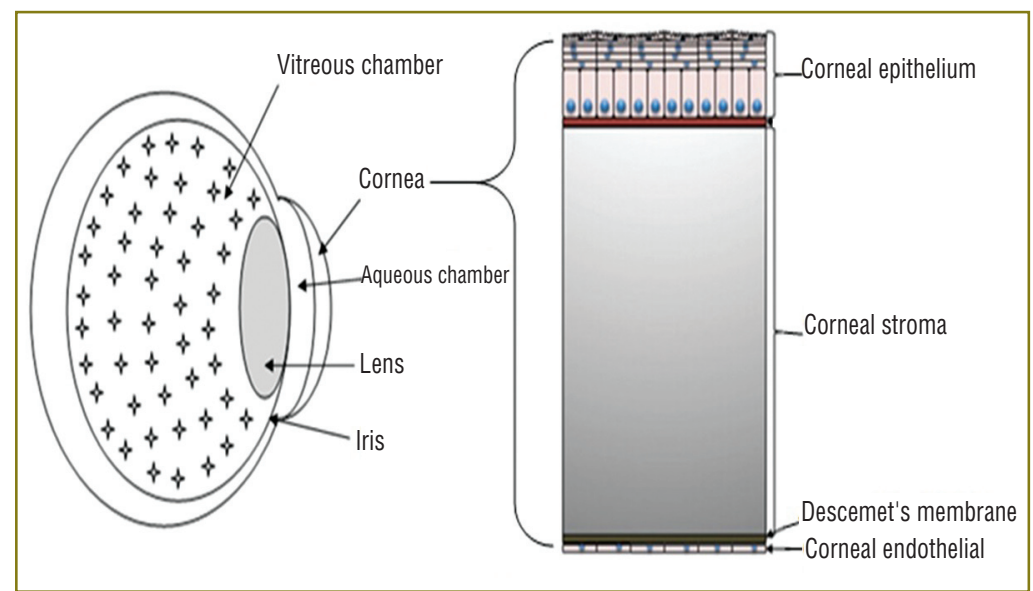

Figure 1. Schematic representation of rabbit cornea. Cross-section of rabbit cornea illustrating the distinct layers of the cornea, i.e. epithelium, corneal stroma and Descemet's membrane 
concentration of collagen filaments is seen throughout the stroma of the rabbit cornea; adjacent to the Descemet's membrane [20].

The rabbit cornea is suitable for our studies for several reasons. Firstly it contains a layered epithelium, separated from an underyling collagen-rich stroma by a basement membrane structure. Hence it contains densely-packed cells of an epithelial lineage on top of a more sparse distribution of fibroblastic cells (keratocytes) within a collagen matrix. This is very similar to the arrangement in most epithelial tissues in the body. Secondly the collagen in cornea is highly organized, leading to very high optical transparency and hence low levels of intrinsic backscatter. This makes it potentially feasible to use OCT to study the depth-resolved propagation of the cellular brightening effect into the stroma, where the cellular signal could otherwise easily be swamped by the much larger signal from disorganized collagen. An advantage of OCT is that it produces robust, quantitative measurements of the tissue backscatter versus depth, with excellent time resolution. Hence we can quantify the degree of cellular backscattering enhancement due to acetic acid, and other substances, in quantified units $(\mathrm{dB})$.

\section{Material and Methods}

Preparation of rabbit cornea. Rabbit eyes obtained from Alison Weather Hook Farm (Hampshire, UK) were used for all experiments. The muscles around the eyes were excised using a sterile scalpel. The eyes were immersed in 3\% iodine for approximately $1 \mathrm{~min}$ and washed in sterile phosphate buffered saline (PBS) to wash off the iodine. All eyes were used immediately after cleaning to minimize degradation of the cornea. The protocol followed is identical to that used in related tissue engineering work in our institute, and allows the extraction of viable epithelial cells which can proliferate in culture [21].

OCT of rabbit cornea. A 4-beam swept source Fourier domain optical coherence tomography system (EX1301, Michelson Diagnostics Ltd., UK) with a swept laser (HSL2000-10, Santec, Inc., Japan) (center wavelength of approximately $1300 \mathrm{~nm}$ and bandwidth of approximately $150 \mathrm{~nm}$ ) was used, giving a two-dimensional cross-sectional visualization of micro-structural morphology at 10,000 Ascans per second. B-scans comprising 952 A-scans are acquired along $4 \mathrm{~mm}$ in lateral direction, at a rate of 5 frames per second.

Throughout our experiments, the OCT system was inverted, i.e. the objective was below the specimen and the beam propagated upwards. The rabbit eyes were placed on top of a slotted ring in a " $\mu$-dish" (a Petri dish with an optical window, $\left.|\mathrm{BID}|^{\circledR}\right)$ with the corneal epithelium facing down towards the optical window and the OCT imaging lens directly below the window. The optical window consists of a microscope cover-slip (approximately $170 \mu \mathrm{m}$ thick), which allowed OCT imaging at around $8 \mu \mathrm{m}$ axial resolution without giving noticeable artefacts. Some slight tilting of the Petri dish was used to attenuate Fresnel reflections from the air-glass interface.

A series of continuous OCT images were taken to study the evolution of aceto-whitening phenomenon. A reference OCT image of the cornea was captured just before topical application of acid. $0.5 \mathrm{M}$ acetic acid was added to the $\mu$-dish just enough to cover the rabbit corneas. Continuous OCT images were taken with a frequency of one image every $4 \mathrm{~s}$ for a total acquisition time of $240 \mathrm{~s}$ with the eyes kept in the same position for the entire time course. The whole protocol was designed to maintain a constant optical arrangement throughout the exposure of the tissue to the acid. This is important because the sensitivity of an OCT system varies significantly if the sample moves relative to the focal point of the objective (confocal roll-off) or relative to the point of zero optical path difference (coherence rolloff). Maintaining a constant optical arrangement ensures that these two effects cannot confound the measured results. This experiment was repeated using $0.5 \mathrm{M}$ lactic acid and PBS as negative controls. Post experiment the rabbit eyes were discarded as per the UK Human Tissue Authority regulations.

OCT data processing. Data acquisition software from Michelson Diagnostics Ltd. (Image4) creates OCT images in PNG format, which normalizes the gray-scale values within the range of 0 to 255 pixels. Additionally the software uses proprietary algorithms to fuse images taken with each of the four independently focused beams. We avoided using these images, instead using a mode of Image4 which records raw digitizer data. Digitized fringe data collected by a single beam were converted into a TIFF image using an in-house MATLAB script, thereby achieving a known, fixed scale for the image pixel intensities in $\mathrm{dB}$ and mapping absolute reflectivities in the range -110 to $-60 \mathrm{~dB}$ on the gray-scale range 0 to 255 . Subsequent analysis of OCT images was performed on these TIFF files.

Quantification of depth-resolved changes in optical properties. Continuous sequences of recorded OCT B-scans were stacked into a video using ImageJ and changes in depth-resolved reflectivity were assessed qualitatively following the addition of acetic acid, lactic acid and PBS.

Quantitative analysis was performed on laterally averaged one-dimensional depth-profiles. The OCT images were first straightened using a straightening algorithm tool in ImageJ [22], so that the corneal surface became flat. Rectilinear regions of interest (ROI) were selected in the straightened OCT image using the rectangular selection tool in ImageJ and were specified to have a lateral dimension of 150 pixels and an axial dimension of 60 pixels (each pixel is of physical size $4.5 \times 4.5 \mu \mathrm{m}$, assuming a tissue refractive index of 1.4). ROl's were selected around areas where a consistent brightening effect was seen in the videos. A laterally averaged depth profile was then generated for the ROI of the straightened OCT image. The reflectivity in $\mathrm{dB}$ at nine different depths $(18,36,54,72,90,135,180$, 125,225 and $270 \mu \mathrm{m}$ ) was then plotted versus time, every $20 \mathrm{~s}$ until $240 \mathrm{~s}$, as well as maximum change in reflectivity over the first $100 \mathrm{~s}$ versus depth.

\section{Results}

Qualitative assessment of OCT images from rabbit cornea. OCT images of rabbit corneas were captured pre and post application of $0.5 \mathrm{M}$ acetic acid (Figure 2 (a) and (b)) and $0.5 \mathrm{M}$ lactic acid (Figure 2 (c) and (d)).

The OCT images before application of either acid shows 

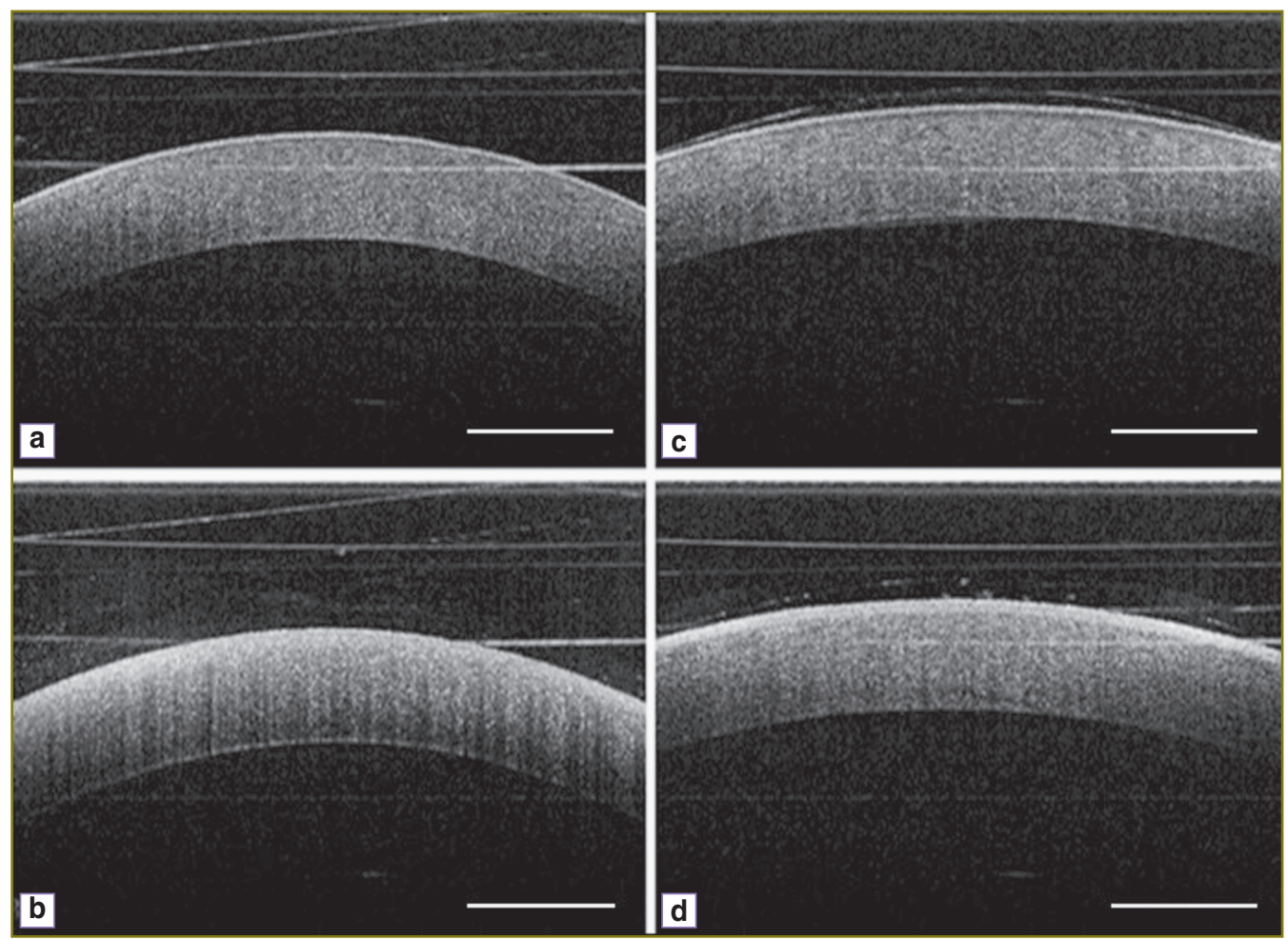

Figure 2. OCT images of rabbit cornea captured using an inverted OCT where the light source is pointing upwards into the objects: (a) before the addition of acetic acid; (b) 4 min after the addition of acetic acid $0.5 \mathrm{M}$; (c) before the addition of lactic acid; (d) 4 min after the addition of lactic acid $0.5 \mathrm{M}$.

Scale bar: $1 \mathrm{~mm}$

a clear demarcation between the corneal epithelium and stroma (See Figure 2 (a) and (c)), whereas after addition of either of the acids this clear demarcation disappeared, due to an increase in reflectivity in the corneal epithelium. The OCT images were taken over a period of $240 \mathrm{~s}$ and the corneas were in contact with the acid for that entire period. Though both acids showed the aceto-whitening effect to be comparable in the epithelium the difference was in the degree of brightness in the corneal stroma post addition of acids. As seen in Figure 2 (b), after exposure to acetic acid the aceto-whitening effect is seen to penetrate into the stroma. In contrast, after exposure to lactic acid (See Figure $2(d)$ ) the effect is limited to approximately $50 \mu \mathrm{m}$ of the total corneal thickness giving rise to a much more distinct narrow white band.

The video sequence of OCT images (Figure 3) clearly shows a rapid brightening effect in the first $100 \mathrm{~s}$, whilst after $100 \mathrm{~s}$ it stays roughly constant. The OCT images $240 \mathrm{~s}$ after acid application showed an overall increase in reflectivity as well as a pattern of vertical dark and bright "banding" (See Figure 3 (a)). This pattern is seen following acetic acid and not lactic acid (See Figure 3 (b)).

Quantitative analysis of one-dimensional reflectivity profiles. Reflectivity profiles corresponding to acetic acid (Figure 4) show that at depths 36 and $54 \mu \mathrm{m}$ the induced reflectivity increase proceeds rapidly, peaking at 11.2 and $8.7 \mathrm{~dB}$ respectively after around $40 \mathrm{~s}$. This is a rapid response however it is broadly in line with the clinically observed AWE timescale [7] and also with the results of mathematical modelling applied to cervical

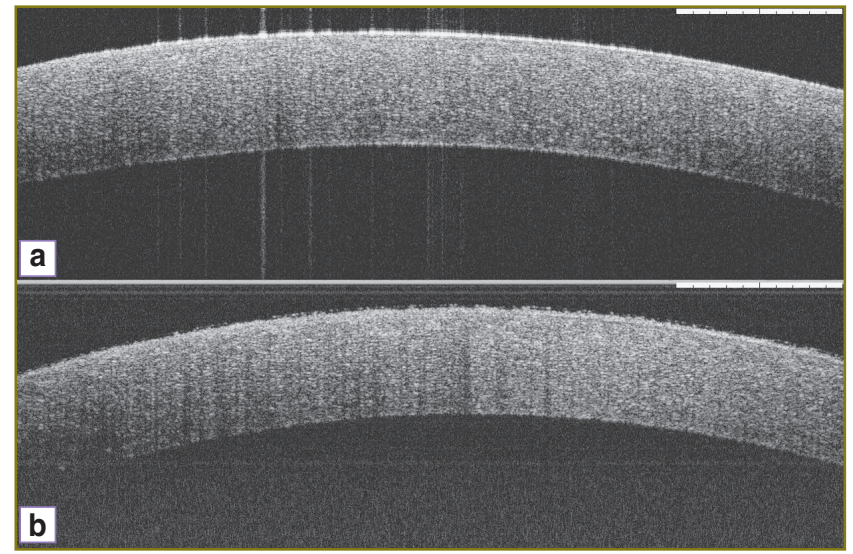

Figure 3. Video sequence showing the propagation of cellular brightening down into rabbit cornea over a total elapsed time of 240 s. Acetic acid (a) is compared directly with lactic acid (b). See Media. Scale bar: $1 \mathrm{~mm}$

epithelium [14]. At depths 72 and $90 \mu \mathrm{m}$, the reflectivity peaks after about $80 \mathrm{~s}$, having increased by about 8.8 and $9.8 \mathrm{~dB}$ respectively. At $135 \mu \mathrm{m}$ depth, it takes $100 \mathrm{~s}$ for the reflectivity to reach a peak of $6 \mathrm{~dB}$. These results show that the deeper the tissue, the longer it takes for the reflectivity to reach a peak. In addition, the greatest change in reflectivity occurs at the more superficial depths, between 36 to $135 \mu \mathrm{m}$. In the first $40 \mathrm{~s}$, the reflectivity between 135 and $270 \mu \mathrm{m}$ decreases significantly. It then recovers over 80 to $100 \mathrm{~s}$. 


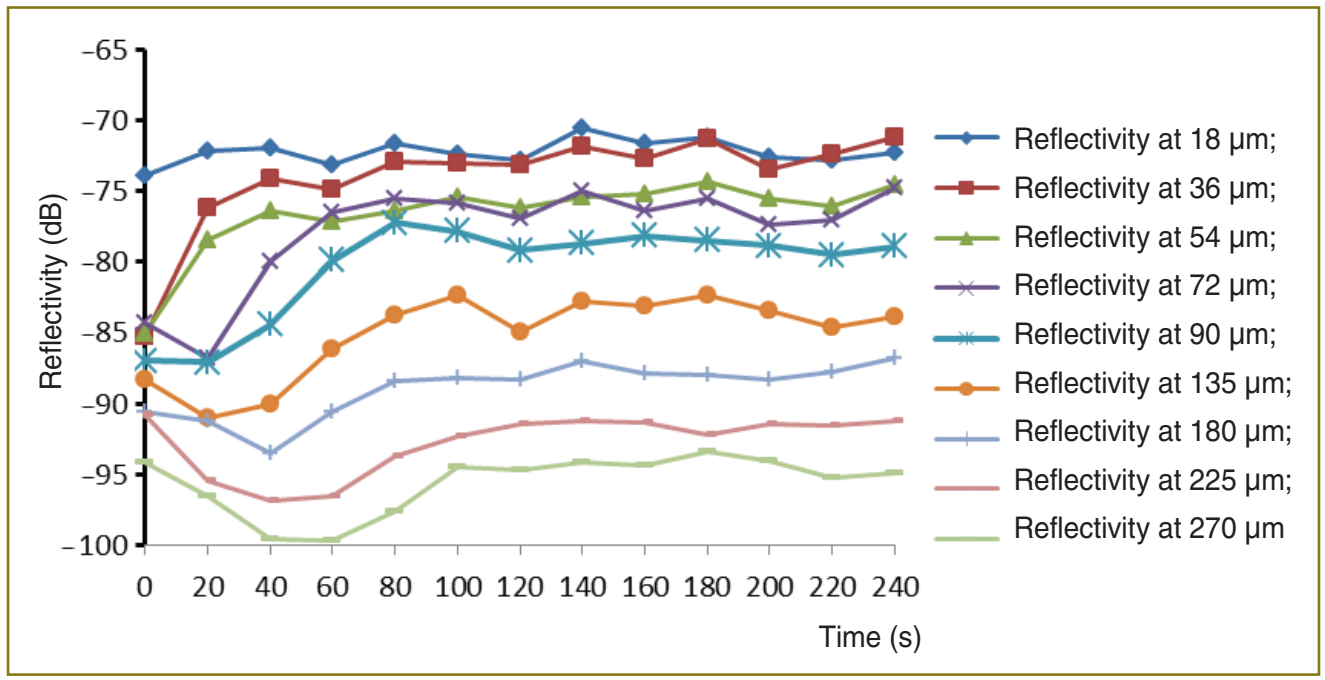

Figure 4. Depth-resolved reflectivity of rabbit cornea with application of $0.5 \mathrm{M}$ acetic acid. Reflectivity curves across different depths of the rabbit cornea after the application of $0.5 \mathrm{M}$ acetic acid

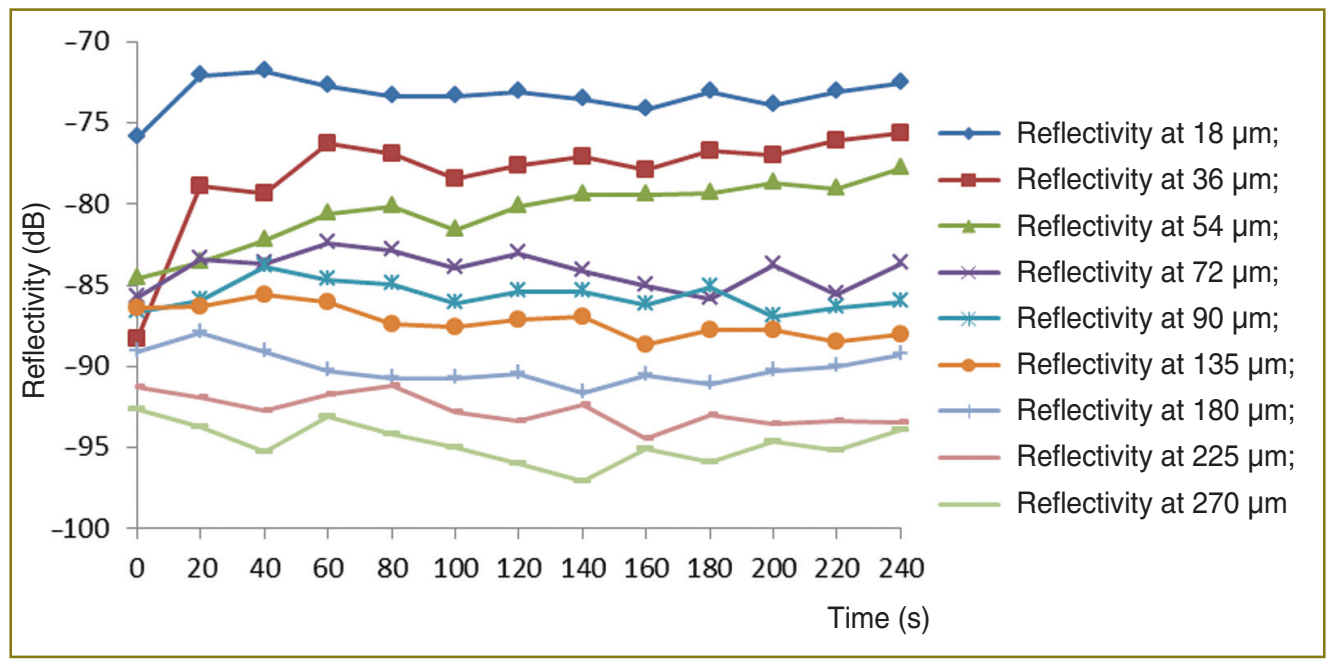

Figure 5. Inverted optical coherence tomography of rabbit cornea with $0.5 \mathrm{M}$ lactic acid. Reflectivity curves across different depths of the rabbit cornea after the application of $0.5 \mathrm{M}$ lactic acid

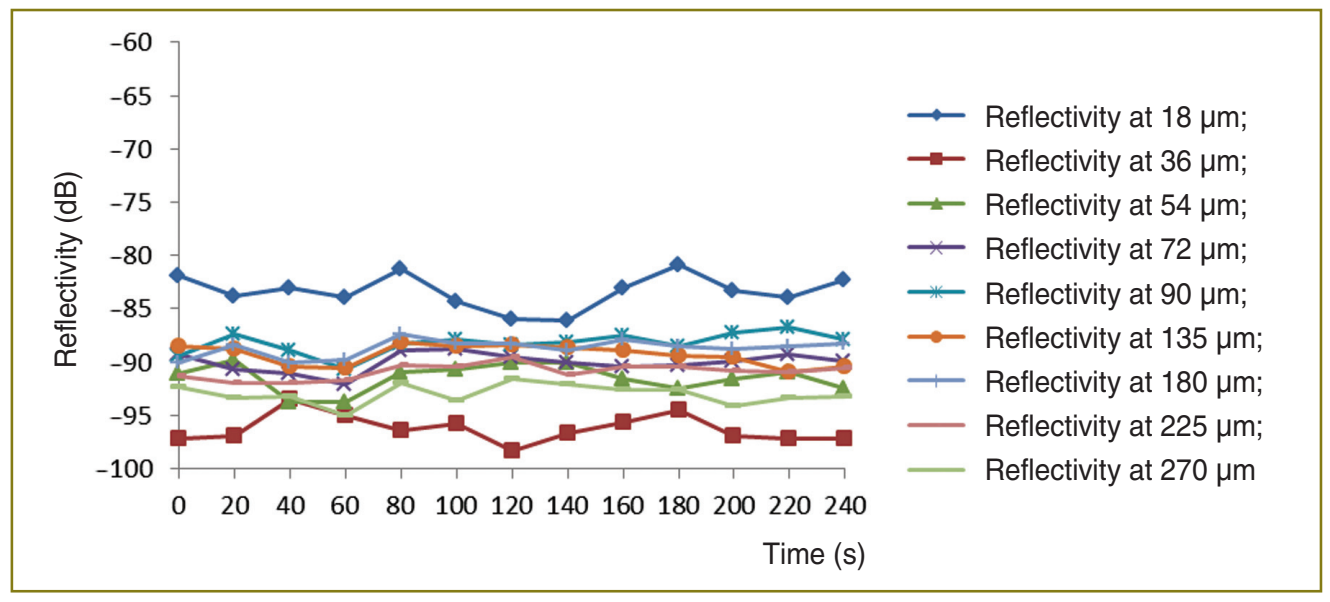

Figure 6. Inverted optical coherence tomography of rabbit cornea with $0.5 \mathrm{ml}$ phosphate buffer solution. Reflectivity curves across the rabbit cornea after the application of phosphate buffer solution 
The results for lactic acid (Figure 5) at superficial depths between 18 and $54 \mu \mathrm{m}$ exhibit a similar pattern to the acetic acid. At $36 \mu \mathrm{m}$ the reflectivity rises rapidly over the first $20 \mathrm{~s}$ and peaks at, having increased by about $12.1 \mathrm{~dB}$. At $54 \mu \mathrm{m}$, the reflectivity peaks at $80 \mathrm{~s}$ and the increase in reflectivity is 3 times smaller than the one at $36 \mu \mathrm{m}$. The reflectivity from depths 72 to $270 \mu \mathrm{m}$ remains stable or falls slightly over $240 \mathrm{~s}$.

Phosphate buffered saline, of physiologically normal tonicity, was used as a second control. The change of reflectivity across different depths of rabbit cornea is shown in Figure 6. At all depths the reflectivity remains roughly constant for the entire $240 \mathrm{~s}$.

The overall change in reflectivity over $240 \mathrm{~s}$ for acetic acid, lactic acid and PBS is tabulated for comparison in the Table. As explained above, a positive change in reflectivity value indicates an increase in the reflectivity whereas a negative value indicates a decrease in the reflectivity. In general, the magnitude of the reflectivity change over $240 \mathrm{~s}$ decreases with depth for both acetic acid and lactic acid. There is a significant drop in reflectivity change between depths 36 to $90 \mu \mathrm{m}$ for acetic acid whereas for lactic acid the intense decline in the reflectivity change occurs more rapidly with depth i.e. between 36 and $54 \mu \mathrm{m}$. There are no significant changes in reflectivity following the application of PBS.

Histology suggests that the epithelial thickness of the rabbit cornea is approximately 35 to $40 \mu \mathrm{m}$. Hence, the epithelial brightening of rabbit cornea is compared for the three substances by plotting the time-course of reflectivity at $36 \mu \mathrm{m}$, as shown Figure 7. Acetic acid and lactic acid exhibit a significant, and broadly comparable, epithelial brightening effect in the first $20 \mathrm{~s}$, with the increase in reflectivity then slowing dramatically. The brightening effect for acetic acid is about $3 \mathrm{~dB}$ greater than for lactic acid. This brightening effect does occur at all for PBS: the reflectivity fluctuates between -93 and $-98 \mathrm{~dB}$.

The brightening after $100 \mathrm{~s}$ versus depth, for acetic acid, lactic acid and PBS is shown in Figure 8. Epithelial brightening (18 to $36 \mu \mathrm{m}$ ), is seen to be broadly comparable for acetic acid and lactic acid but absent for PBS. The major distinction between acetic acid and lactic acid occurs over

\section{Overall change in reflectivity across different depths of rabbit cornea}

\begin{tabular}{|cccc|}
\hline $\begin{array}{c}\text { Depth of rabbit } \\
\text { Cornea }(\boldsymbol{\mu m})\end{array}$ & \multicolumn{2}{c|}{ Overall change in reflectivity over $\mathbf{2 4 0 ~} \mathbf{~ ( d B )}$} \\
\hline 36 & $\begin{array}{c}\mathbf{0 . 5} \mathbf{M} \\
\text { acetic acids }\end{array}$ & $\begin{array}{c}\mathbf{0 . 5} \mathbf{~ M} \\
\text { lactic acids }\end{array}$ & $\begin{array}{c}\text { Phosphate buffer } \\
\text { solutions }\end{array}$ \\
\hline 54 & 14.1 & 12.7 & 0.1 \\
\hline 72 & 10.6 & 6.8 & -1.4 \\
\hline 90 & 9.6 & 2.1 & -0.6 \\
\hline 135 & 8.1 & 0.6 & 1.54 \\
\hline 180 & 4.5 & -1.6 & -1.92 \\
\hline 225 & 3.8 & -0.2 & 1.78 \\
\hline 270 & -0.4 & -2.2 & 0.86 \\
\hline
\end{tabular}

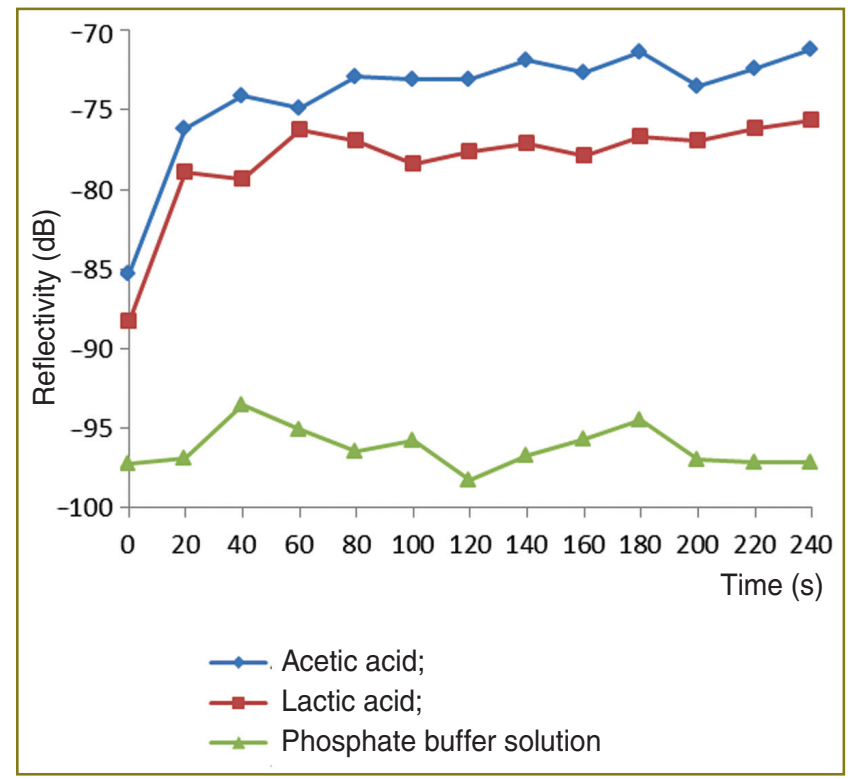

Figure 7. Epithelial brightening of rabbit cornea at $36 \mu \mathrm{m}$ depth after the application of acetic acid, lactic acid and phosphate buffer solution

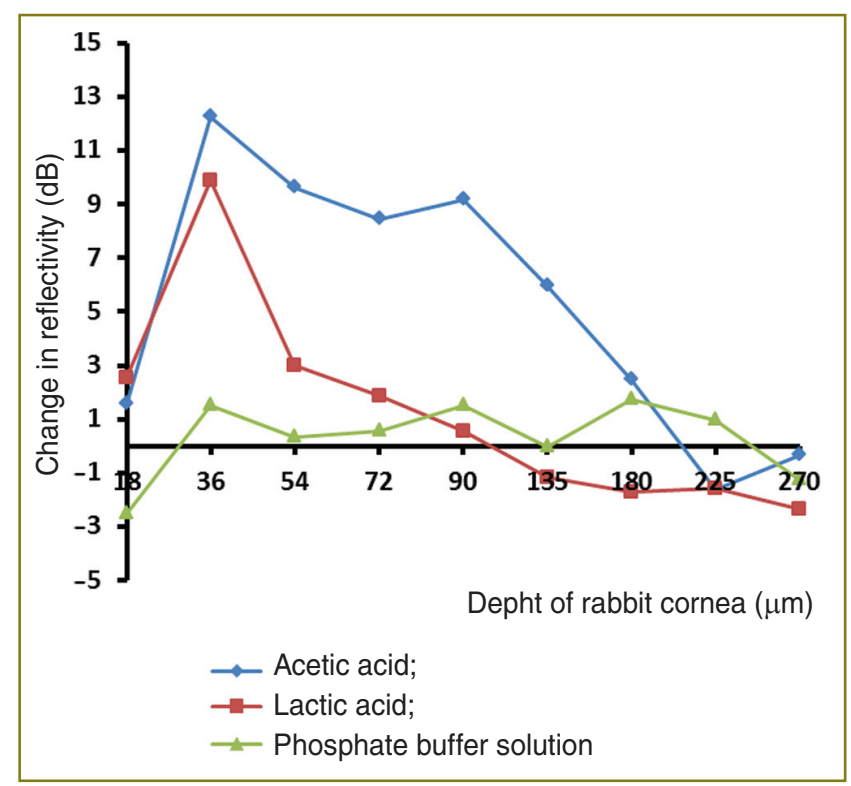

Figure 8. Depth-resolved brightening of the rabbit cornea after $100 \mathrm{~s}$, as produced by acetic acid, lactic acid and phosphate buffer solution

the depth ranges beyond $36 \mu \mathrm{m}$, i.e. the stromal layers. Here the brightening magnitude drops drastically for lactic acid but remains significant for acetic acid down to depths approaching $200 \mu \mathrm{m}$.

Discussion. The use of rabbit cornea in combination with an inverted optical coherence tomography microscope system provided stable and reproducible measurements of the depth-resolved changes in tissue backscatter due to the addition of acetic acid, lactic acid and PBS.

For acetic acid, OCT readily demonstrated a propagating wave of tissue brightening moving down through the 
epithelium and into the stroma. The time-resolved brightening curve generally showed a rapid initial phase, followed by a much slower late phase. The time scale of the initial phase increased from around $30 \mathrm{~s}$ at $36 \mu \mathrm{m}$ depth to around $80 \mathrm{~s}$ at $90 \mu \mathrm{m}$ depth. The brightening did not show a strong spatial localization but instead appeared to "wash through" the epithelium and into the stroma. The magnitude of the brightening effect fell with depth, having a peak value of around $12 \mathrm{~dB}$ near the surface. Patel demonstrated that the rabbit keratocytes density decreases significantly from the corneal epithelium through the stroma [23]. This suggests that acetic acid has a significant optical effect both on corneal epithelial cells and on fibroblastic keratocytes. An interesting late-stage optical effect of acetic acid is the appearance of a pattern of dark vertical stripes in a single B-scan. The stripes appeared to begin in the stroma. The origin of this pattern was not determined but presumably is generated by an optical shadowing effect caused by a large change in scattering properties in structures at the top of each stripe. The phenomenon was not seen with either lactic acid nor PBS.

Phosphate buffered saline behaved as we expected in that it acted as a negative control for cellular brightening. Hypertonic saline $(840 \mathrm{mM}$, i.e. $\times 6$ physiological concentration) has been reported to increase the backscatter from cytoplasm relative to nuclear material when imaged by $808 \mathrm{~nm}$ confocal microscopy [5], however we found no significant effect using PBS at physiological concentration with $1300 \mathrm{~nm}$ OCT.

The most unexpected result from our study was the behavior of lactic acid. This alpha-hydroxy acid has been reported as a negative control for clinical AWE [7], however we found that it produces brightening in the surface epithelium of up to $12 \mathrm{~dB}$, which is very similar to the effect of acetic acid. The main difference with acetic acid lay in the depth resolved kinetics of the brightening. Whereas acetic acid appeared to wash through the epithelium and into the stroma, the lactic acid produced a clearly demarcated zone of brightening within the surface $30 \mu \mathrm{m}$ or so. We postulate that this surface zone corresponds to the corneal epithelium since the thickness is in good agreement with literature values.

Our results have implications for research into the origins of clinical AWE and the wider area of the control of biological tissue scattering. It is generally assumed that the AWE arises in the epithelium, however this conclusion seems at odds with our observation that lactic acid brightens corneal epithelial cells by a similar amount to acetic acid whereas lactic acid does not produce clinical AWE. It may be that the brightening effect of acetic acid is highly specific to the sub-type of epithelial cell. Or the corneal epithelium model may not capture certain key parameters (possibly baseline $\mathrm{pO}_{2}$, baseline $\mathrm{pH}$, squamous vs columnar cell phenotype etc) that are required for clinical AWE but instead merely displays a sub-clinical effect, in which case model systems designed to study the effect may need to be further refined.

The strong confinement of cellular brightening to the surface epithelium displayed by lactic acid is also intriguing. We did not determine whether this was caused by an inability of the lactic acid to penetrate through to the stroma (e.g. by being blocked by the basal lamina) or due to a lack of optical interaction with the fibroblastic keratocytes. The former explanation seems unlikely as both acids are very low molecular weight (60 vs $90 \mathrm{Da}$ for acetic and lactic respectively). The latter explanation is intriguing in the context of Maddox et al. [8] hypothesis concerning the role of cytokeratins in acetowhitening. Cytokeratins may be expected to be present in the corneal epithelial cells but absent in the stromal keratocytes. More studies are needed to determine the merits of these, or other, hypotheses.

In conclusion, we have shown for the first time that OCT can provide direct measurements of the deph-resolved kinetics of the aceto-whitening phenomenon in a 3D epithelial tissue. This data can give important guidance to quantitative models of the phenomenon. We have also shown, for the first time to our knowledge, that cells of different phenotypes can be discriminated based on their response to acetic vs lactic acid. Our results show that the phenomenon of aceto-whitening, and the more general field of biological scattering control, continues to pose interesting research questions.

Study Funding. EPSRC grants EP/EI018328, EP/ F020422.

Conflict of Interest. The authors have no conflict of interests to disclose.

\section{References}

1. Collier T., Lacy A., Richards-Kortum R., Malpica A., Follen M. Near real-time confocal microscopy of amelanotic tissue: detection of dysplasia in ex vivo cervical tissue. Acad Radiol 2002; 9(5): 504-512, http://dx.doi.org/10.1016/s10766332(03)80326-4.

2. Hoffman A., Goetz M., Vieth M., Galle P.R., Neurath M.F., Kiesslich R. Confocal laser endomicroscopy: technical status and current indications. Endoscopy 2006; 38(12): 1275-1283, http://dx.doi.org/10.1055/s-2006-944813.

3. Zysk A.M., Nguyen F.T., Oldenburg A.L., Marks D.L., Boppart S.A. Optical coherence tomography: a review of clinical development from bench to bedside. J Biomed Opt 2007; 12(5): 051403, http://dx.doi.org/10.1117/1.2793736.

4. Coleman A.J., Richardson T.J., Orchard G., Uddin A., Choi M.J., Lacy K.E. Histological correlates of optical coherence tomography in non-melanoma skin cancer. Skin Res Technol 2013; 19(1): e10-e19, http://dx.doi.org/10.1111/ j.1600-0846.2012.00626.x.

5. Pierce M.C., Javier D.J., Richards-Kortum R. Optical contrast agents and imaging systems for detection and diagnosis of cancer. Int J Cancer 2008; 123(9): 1979-1990, http://dx.doi.org/10.1002/ijc.23858.

6. Xia Y., Hallas N.J. Shape-controlled synthesis and surface plasmonic properties of metallic nanostructures. MRS Bulletin 2005; 30(5): 338-348, http://dx.doi.org/10.1557/ mrs2005.96.

7. MacLean A.B. Acetowhite epithelium. Gynecol Oncol 2004; 95(3): 691-694, http://dx.doi.org/10.1016/ j.ygyno.2004.08.006.

8. Maddox P., Szarewski A., Dyson J., Cuzick J. Cytokeratin expression and acetowhite change in cervical 
epithelium. J Clin Pathol 1994; 47(1): 15-17, http://dx.doi. org/10.1136/jcp.47.1.15.

9. Marina O.C., Sanders C.K., Mourant J.R. Effects of acetic acid on light scattering from cells. J Biomed Opt 2012; 17(8): 085002, http://dx.doi.org/10.1117/1.jbo.17.8.085002.

10. Longcroft-Wheaton G., Duku M., Mead R., Poller D., Bhandari P. Acetic acid spray is an effective tool for the endoscopic detection of neoplasia in Barrett's esophagus. Clin Gastroenterol Hepatol 2010; 8(10): 843-847, http://dx.doi. org/10.1016/j.cgh.2010.06.016.

11. Yagi K., Aruga Y., Nakamura A., Sekine A., Umezu $H$. The study of dynamic chemical magnifying endoscopy in gastric neoplasia. Gastrointest Endosc 2005; 62(6): 963-969, http://dx.doi.org/10.1016/j.gie.2005.08.050.

12. Kawahara Y., Takenaka R., Okada H., Kawano S., Inoue M., Tsuzuki T., Tanioka D., Hori K., Yamamoto K. Novel chromoendoscopic method using an acetic acid-indigocarmine mixture for diagnostic accuracy in delineating the margin of early gastric cancers. Dig Endosc 2009; 21(1): 14-19, http:// dx.doi.org/10.1111/j.1443-1661.2008.00824.x.

13. Bhalang K., Suesuwan A., Dhanuthai K., Sannikorn P., Luangjarmekorn L., Swasdison S. The application of acetic acid in the detection of oral squamous cell carcinoma. Oral Surg Oral Med Oral Pathol Oral Radiol Endod 2008; 106(3): 371-376, http://dx.doi.org/10.1016/j.tripleo.2008.01.017.

14. Balas C., Papoutsoglou G., Potirakis A. In vivo molecular imaging of cervical neoplasia using acetic acid as biomarker. IEEE J Select Topics Quantum Electron 2008; 14(1): 29-42, http://dx.doi.org/10.1109/jstqe.2007.913396.

15. Balas C. A novel optical imaging method for the early detection, quantitative grading and mapping of cancerous and precancerous lesions of cervix. IEEE Trans Biomed Eng 2001; 48(1): 96-104, http://dx.doi.org/10.1109/10.900259.

16. van Praag E.V. Corneal abrasion and ulceration in rabbits. Available: http://www.medirabbit.com/EN/Eye_ diseases/Disorder/Ulcer/Ulc_en.htm.

17. Du L., Wu X., Pang K., Yang Y. Histological evaluation and biomechanical characterisation of an acellular porcine scaffold. Br J Opthalmol 2011; 95(3): 410-414, http://dx.doi. org/10.1136/bjo.2008.142539.

18. Hahne M., Reichl S. Development of a serum-free human cornea construct for in vitro drug absorption studies: the influence of varying cultivation parameters on barrier characteristics. Int J Pharm 2011; 416(1): 268-279, http:// dx.doi.org/10.1016/j.ijpharm.2011.07.004.

19. Masters B.R., Paddock S. In vitro confocal imaging of rabbit cornea. J Microsc 1990; 158(2): 267-274, http://dx.doi. org/10.1111/j.1365-2818.1990.tb03000.x.

20. Ojeda J.L., Ventosa J.A., Piedra S. The threedimensional microanatomy of the rabbit and human cornea. A chemical and mechanical microdissection-SEM approach. J Anat 2001; 199(5): 567-576, http://dx.doi.org/10.1046/ j.1469-7580.2001.19950567.x.

21. Deshpande P., Notara M., Bullett N., Daniels J.T., Haddow D.B., MacNeil S. Development of a surface-modified contact lens for the transfer of cultured limbal epithelial cells to the cornea for ocular surface diseases. Tissue Eng Part A 2009; 15(10): 2889-2902, http://dx.doi.org/10.1089/ten. TEA.2008.0528.

22. Kocsis E., Trus B.L., Steer C.J., Bisher M.E., Steven A.C. Image averaging of flexible fibrous macromolecules: the clathrin triskelion has an elastic proximal segment. J Struct Biol 1991; 107(1): 6-14, http://dx.doi.org/10.1016/10478477(91)90025-r.

23. Patel S.V., McLaren J.W., Camp J.J., Nelson L.R., Bourne W.M. Automated quantification of keratocyte density by using confocal microscopy in vivo. Invest Opthalmol Vis Sci 1999; 40(2): 320-336. 